\title{
Aberrant perichondrial BMP signaling mediates multiple osteochondromagenesis in mice
}

\author{
Toshihiro Inubushi, Satoshi Nozawa, Kazu Matsumoto, Fumitoshi Irie, and Yu Yamaguchi \\ Human Genetics Program, Sanford Burnham Prebys Medical Discovery Institute, La Jolla, California, USA.
}

\begin{abstract}
Multiple hereditary exostoses (MHE) is characterized by the development of numerous benign bony tumors (osteochondromas). Although it has been well established that MHE is caused by mutations in EXT1 and EXT2, which encode glycosyltransferase essential for heparan sulfate (HS) biosynthesis, the cellular origin and molecular mechanisms of MHE remain elusive. Here, we show that in Ext1 mutant mice, osteochondromas develop from mesenchymal stem cell-like progenitor cells residing in the perichondrium, and we show that enhanced BMP signaling in these cells is the primary signaling defect that leads to osteochondromagenesis. We demonstrate that progenitor cells in the perichondrium, including those in the groove of Ranvier, highly express HS and that Ext1 ablation targeted to the perichondrium results in the development of osteochondromas. Ext1deficient perichondrial progenitor cells show enhanced BMP signaling and increased chondrogenic differentiation both in vitro and in vivo. Consistent with the functional role for enhanced BMP signaling in osteochondromagenesis, administration of the small molecule BMP inhibitor LDN193189 suppresses osteochondroma formation in two MHE mouse models. Together, our results demonstrate a role for enhanced perichondrial BMP signaling in osteochondromagenesis in mice, and they suggest the possibility of pharmacological treatment of MHE with BMP inhibitors.
\end{abstract}

Conflict of interest: $Y Y$ is a recipient of a research grant from Clementia Pharmaceuticals.

Submitted: September 12, 2016

Accepted: June 23, 2017

Published: August 3, 2017

\section{Reference information:}

JCI Insight. 2017;2(15):e90049.

https://doi.org/10.1172/jci.

insight.90049.

\section{Introduction}

Heparan sulfate (HS) is a sulfated glycosaminoglycan found on cell surfaces and in the extracellular matrix. HS is functionally involved in diverse developmental processes. Endochondral ossification is one of such developmental processes in which HS plays a particularly important role (1). Multiple hereditary exostoses (MHE) is a genetic bone disorder caused by heterozygous loss-of-function mutations in EXT1 or EXT2, both of which are required for HS biosynthesis. It is one of the most common inherited skeletal dysplasias in humans, with an estimated prevalence of at least 1 in 50,000 (2). EXT1 encodes the key $N$-acetylglucosamine/glucuronic acid copolymerase for elongation of the HS backbone, while EXT2 encodes a protein that associates with EXT1 protein and transports it to the Golgi apparatus, where HS synthesis occurs $(3,4)$. Homozygous ablation of either gene results in essentially complete abrogation of HS production in cells, while heterozygous cells, including somatic cells in patients of MHE, generally express HS at approximately half the level of WT cells $(5,6)$. MHE is characterized by the development of multiple cartilage-capped, bony protrusions, which are histologically defined as osteochondromas. Osteochondromas in MHE develop in bones of endochondral origin, such as long bones, rib bones, scapula, and vertebrae (7).

We previously demonstrated that stochastic inactivation of Ext1 in a small fraction of Col2a1-expressing cells using a Col2a1-Cre ${ }^{E R T}$ transgene (8) and the loxP-modified allele Ext $1^{f l o x}$ (9) (Col2a1-Cre ${ }^{E R T}$;Ext $1^{f l o x} / f l o x$ mice, called Col2a1-Ext ${ }^{\text {CKO }}$ mice hereafter) results in the development of multiple osteochondromas and skeletal deformities in mice that closely resemble the human MHE phenotype in mice (10). Jones et al. (11) independently made essentially the same observation using a different genetic mouse model. These results point to the involvement of loss of heterozygosity in the pathogenesis of human MHE. Furthermore, the level of phenotypic recapitulation and the experimental tractability of the Col2a1-Ext $1^{\text {CKO }}$ stochastic KO model make it very useful for studying MHE.

While the previous knockout studies $(10,11)$ have provided a clue to the genetic mechanism of MHE, the molecular and cellular mechanisms that lead to the development of multiple bone tumors remain elusive. 
In this paper, we sought to address two questions concerning the pathogenesis of MHE, namely the cellular origin of osteochondromas and the signaling aberration underlying osteochondromagenesis. To address the former question, we generated a MHE mouse model based on Fsp1-Cre-mediated conditional KO, in which Ext1 was ablated in progenitor cells in the perichondrium but not in chondrocytes in the growth plate. We show that Fsp1-Cre;Ext $1^{f l o x / f l o x}$ mice (called Fsp1-Ext $1^{C K O}$ mice, hereafter) developed multiple osteochondromas, demonstrating that osteochondromas can be derived from Ext1-deficient progenitor cells in the perichondrium. As for the signaling aberration underlying osteochondromagenesis, we show that BMP signaling is upregulated in the perichondrium of both Col2a1-Ext $1^{\text {CKO }}$ and Fsp1-Ext ${ }^{\text {CKO }}$ mice, and that Ext1-deficient perichondrial progenitor cells exhibit enhanced chondrogenic differentiation and BMP signaling in vitro. Furthermore, pharmacological intervention of BMP signaling using the small molecule BMP inhibitor LDN-193189 suppresses the development of osteochondromas and bone deformity in these two MHE models. Overall, our results demonstrate that aberrant perichondrial BMP signaling in progenitor cells in the perichondrium mediates osteochondromagenesis in mice, and they suggest a potential therapeutic and/or preventive role for BMP inhibitors in the treatment of $\mathrm{MHE}$

\section{Results}

Aberrant differentiation of perichondrial cells in Col2a1-Ext $1^{\text {CKO }}$ mice. We have previously shown that Col2a1-Ext $1^{\text {CKO }}$ mice develop macroscopic osteochondromas in various bones by P28 (10). To identify earlier changes associated with osteochondroma development, we histologically examined the wrist joint area of Col2a1-Ext1 ${ }^{\text {CKO }}$ mice at P10, where osteochondromas develop at 100\% penetrance by P28 (10). Safranin O staining revealed the presence of abnormal cell clusters in the groove of Ranvier, a specialized area of the perichondrium circumferencing the epiphyseal growth plate (Figure 1A). No such abnormalities were observed in the groove of Ranvier of control mice (Figure 1A). Similar abnormal cell clusters were also observed in the perichondrium of rib bones of Col2a1-Ext1 ${ }^{\text {CKO }}$ mice (Figure 1B). These observations suggest that osteochondromas are derived from aberrant differentiation of cells in the perichondrium, as postulated previously (12-14).

The groove of Ranvier is thought to serve as a stem cell niche for generating cells of chondrogenic and osteogenic lineages (15). We confirmed that cells in the groove of Ranvier express mesenchymal stem cell (MSC) markers, CD44, Sca-1, and Stro-1 (Figure 1C). The presence of high levels of HS in the perichondrium has been suggested by a previous in situ hybridization study, which showed robust expression of mRNA encoding the syndecan-3 core protein in the perichondrium of developing chick bones (16). To directly examine the presence of HS in the perichondrium, we used two monoclonal antibodies (10E4 and 3G10; ref. 17) that recognize different carbohydrate epitopes in HS chains. The groove of Ranvier is strongly immunoreactive to both antibodies (Figure 1C and Supplemental Figure 1; supplemental material available online with this article; https://doi.org/10.1172/jci.insight.90049DS1). Specificity of this result was further confirmed by using heparitinase treatment of tissue sections (Supplemental Figure 1). HS immunoreactivity is detectable in growth plate cartilage, but its intensity is much weaker than that of the perichondrium (Figure 1C). These results demonstrate that, in the epiphyseal area of developing long bones, HS is predominantly expressed in the perichondrium, including the groove of Ranvier, suggesting its possible functional involvement in the proliferation and/or differentiation of progenitor cells.

To determine the differentiation state of the abnormal cell clusters, we performed double immunostaining with antibodies to Sox9 (a marker of early chondrogenic cells) and type II collagen (a marker for differentiated chondrocytes). In control mice, the distribution of Sox9-expressing cells (green) was restricted to growth plate cartilage; no Sox9-expressing cells were detected in the perichondrium (Figure 1D). In contrast, in Col2a1-Ext ${ }^{C K O}$ mice, cells ectopically expressing Sox9 were detected in the perichondrium, coinciding with the groove of Ranvier (Figure 1D, white arrowheads). Similarly, ectopic Sox9-expressing cells

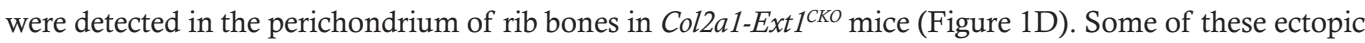
Sox9-expressing cells elaborate pericellular coats of type II collagen (Figure 1D, open arrowhead). To further characterize the property of these cells, sections through Sox9-expressing cell clusters were analyzed for MSC marker expression. Immunostaining with anti-CD44 antibody revealed that these cells express CD44, albeit at lower levels than surrounding normal perichondrial cells (Figure 1E), suggesting that they arise from progenitor cells. Together, these results suggest that loss of HS causes premature chondrogenic differentiation of progenitor cells in the perichondrium.

Perichondrium-targeted conditional Ext1 deletion leads to osteochondroma formation. The identification of abnormal cell clusters in the groove of Ranvier of Col2a1-Ext $1^{\text {CKO }}$ mice suggests that osteochondromas are derived 
A
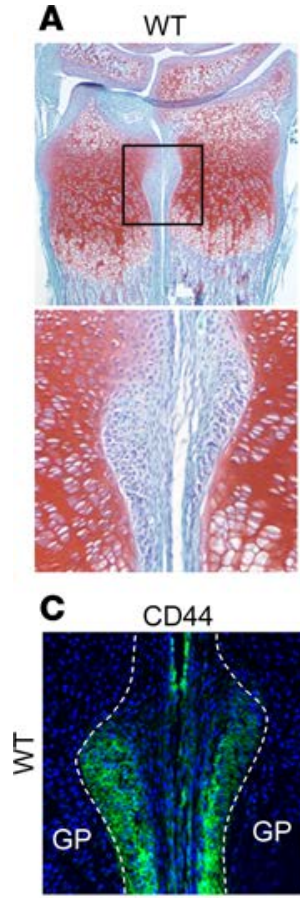

D
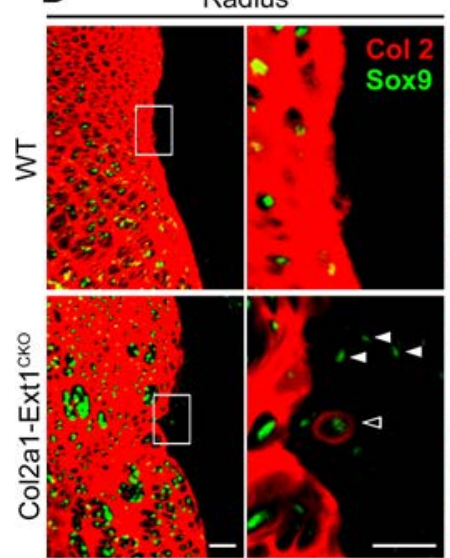

Col2a1-Ext1 ${ }^{\text {cko }}$

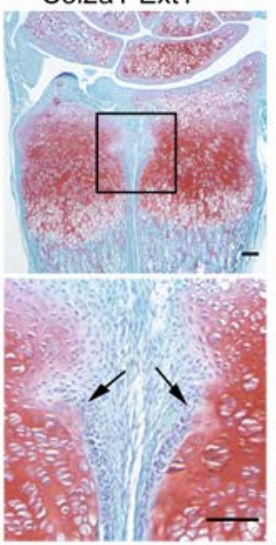

Sca-1
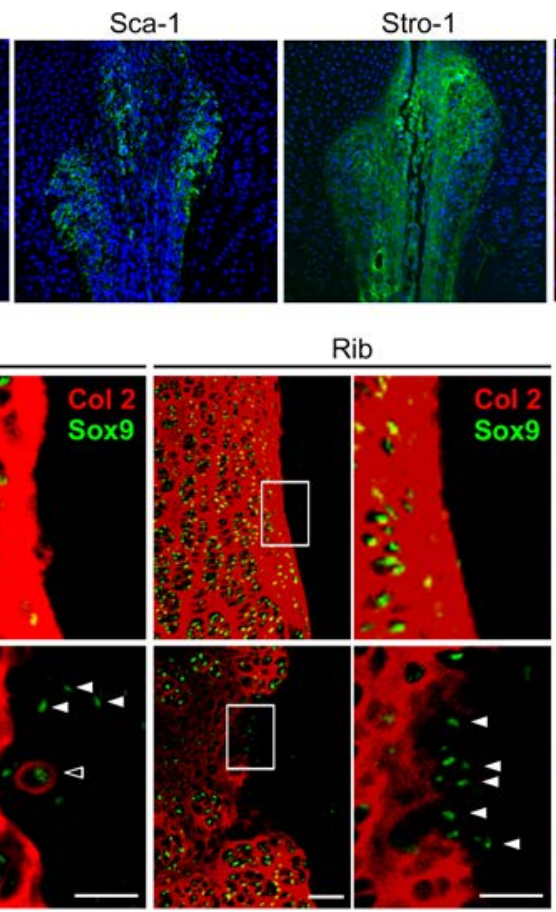

Rib

B
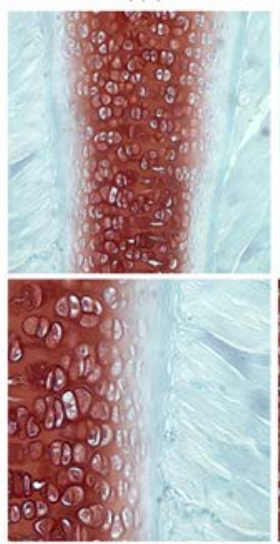

Stro-1

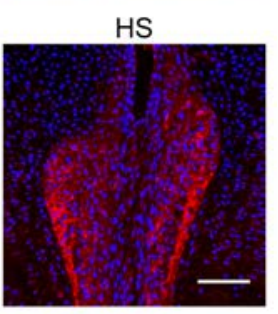

E

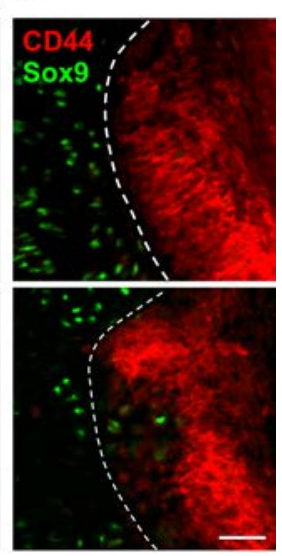

Col2a1-Ext1 ${ }^{\text {cko }}$
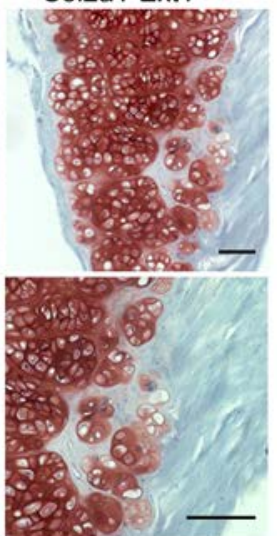

HS
Figure 1. Aberrant differentiation of perichondrial cells in Col2a1-Ext ${ }^{\mathrm{CKO}}$ mice. (A) Sections of the forelimb of Col2a1-Ext1 ${ }^{\text {CKO }}$ mice and their control littermates (Ext7flox/flox; WT) at P10 stained with Safranin O/Fast Green. Lower panels show enlarged views of the outlined areas. Note that the smooth cartilage/perichondrium boundary in WT mice is disorganized in Col2a1-Ext1 ${ }^{\text {CKO }}$ mice, and that the abnormal clusters of weakly Safranin 0-stained cells are present in the groove of Ranvier (arrows). (B) Longitudinal sections of a rib bone of Col2a1-Ext1 $1^{\text {KKO }}$ and control (Ext7 flox/flox; WT) mice at P10 stained with Safranin 0/Fast Green. The cartilage/perichondrium boundary is severely disorganized in Col2a1-Ext ${ }^{\tau K O}$ mice, and abnormal cell clusters are present in the cartilage/perichondrium boundary. (C) Immunohistochemical staining of the perichondrial groove of Ranvier in P10 WT mice for mesenchymal stem cell (MSC) markers (CD44, Sca-1, Stro-1) and heparan sulfate (HS). (D) Immunohistochemical characterization of perichondrial cells in Col2a1-Ext ${ }^{\text {CKO }}$ and control (Ext flox/flox; WT) mice at P10. Sections of the radius and a rib bone were stained with anti-Sox9 and anti-type II collagen (Col2) antibodies. Areas indicated by rectangles are enlarged in neighboring panels. Note the presence of ectopic Sox9-expressing cells in the mutant perichondrium (white arrowheads). Some of these cells also elaborate type II collagen pericellularly (open arrowheads). (E) Characterization of Sox9-expressing ectopic cell clusters in the groove of Ranvier by double-staining with anti-Sox9 and anti-CD44 antibodies. Note that ectopic Sox9-expressing cells in the mutant perichondrium are weakly immunoreactive to antiCD44. Scale bars: $0.1 \mathrm{~mm}$. Broken lines in $\mathbf{C}$ and $\mathbf{E}$ indicate the cartilage/perichondrium boundary. GP, growth plate. Data shown are representative images; each analysis was performed on at least 3 animals per genotype.

from progenitor cells residing in the perichondrium. Although Col2al is generally considered to be a marker for differentiated chondrocytes, Col2a1-Cre has been shown to be active in the perichondrium $(8,18,19)$. Therefore, the development of osteochondromas in Col2a1-Ext1 ${ }^{C K O}$ mice does not rule out the possibility that they are derived from cells in the perichondrium. To obtain genetic evidence for this possibility, we generated perichondrium-targeted Ext1-conditional KO mice using Fsp1-Cre (20). Fsp1 is expressed by precursor cells of the mesenchymal lineage but not by terminally differentiated mesenchymal cell types, such as chondrocytes or osteocytes (21-23), and its expression in developing bone is restricted to the perichondrium and periosteum (24). The Fsp1-Cre transgene has previously been used to ablate Ptpn11 in the perichondrium (25). The recombination pattern of Fsp1-Cre, visualized using the Rosa26-lacZ $(R 26 R)$ reporter, demonstrates that its activity in developing bone is restricted to the perichondrium and periosteum (Supplemental Figure 2).

Fsp1-Ext1 ${ }^{\text {CKO }}$ mice were born alive without any gross abnormalities, and the overall skeletal development of these mice was indistinguishable from control littermates. At 4 weeks, bony protrusions were identified in limb, rib, and vertebral bones (Figure 2, A and B). Histologically, these protrusions consist of bony tuberosities with a cartilage cap (Figure 2C), consistent with the histological features of osteochondromas in human MHE. These mice also displayed rib-cage deformity and swelling of the chondro-osseous junction, phenotypes also observed in Col2a1-Ext1 СКО mice (10). Supplemental Table 1 summarizes the occurrence of osteochondromas in Fsp1-Ext $1^{\text {CKO }}$ mice at 4 weeks of age in comparison with Col2a1-Ext $1^{\text {CKO }}$ 
mice (10). The penetrance of the phenotypes is largely comparable between the two models, except for the higher occurrence of osteochondromas in the distal humerus, proximal radius, and distal femur and

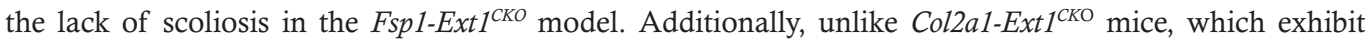
enhanced osteoclastogenesis (26), there was no increase in the number of osteoclasts in Fsp1-Ext ${ }^{\text {CKO }}$ mice (Supplemental Figure 3). Overall, these results demonstrate that loss of Ext1 restricted to perichondrial progenitor cells is sufficient to induce MHE-like osteochondromagenesis in various bones.

Ext1-deficient perichondrium-derived progenitor cells exhibit enhanced chondrogenic differentiation and BMP signaling. While HS plays a role in multiple signaling pathways, the demonstration that the abnormal cell clusters in the perichondrium express Sox 9 and Col2 suggests that the pathway primarily affected in the course of osteochondromagenesis is one involved in chondrogenic differentiation. One such candidates is the BMP pathway, which is noteworthy in that several lines of evidence suggest a functional link between BMP signaling and HS in the context of endochondral ossification. For example, conditional inactivation of Ext1 in the limb bud mesenchyme primarily affects the BMP pathway by inducing the spatial expansion of BMP signaling (10). Both enzymatic removal of HS and chemical interference with HS enhance BMP bioactivity $(12,27,28)$. Transgenic mice expressing a constitutively active form of the BMP receptor 1A develop abnormal chondrocytic protrusions in the perichondrium (29). Most intriguingly, patients of fibrodysplasia ossificans progressiva (FOP), which is caused by a constitutively active mutation of the type I BMP receptor ALK2, have been reported to develop osteochondroma-like bone protrusions with a high frequency (30). These observations suggest that aberrant BMP signaling in HS-deficient cells in the perichondrium is a key molecular culprit underlying osteochondromagenesis.

To examine this possibility, we employed primary cultures of perichondrium-derived mesenchymal progenitor cells $(31,32)$ (designated as PDPCs). The isolated cells express MSC markers (Supplemental Figure $4 \mathrm{~A}$ ) and are capable of differentiation along the adipogenic, chondrogenic, and osteogenic lineages (Supplemental Figure 4B). Ext1-deficient and control PDPCs were prepared by infecting Ext $1^{\text {flox/flox }}$ PDPCs with lentivirus-expressing Cre (lenti-Cre) or insertless lentivirus (lenti-control), respectively. Near complete ablation of Ext1 and HS expression in lenti-Cre-infected cells was confirmed by IHC with the 10E4 antiHS antibody (Supplemental Figure 4C) and quantitative PCR (qPCR) of Ext1 (Supplemental Figure 4D).

In pellet cultures, Ext1-deficient PDPCs showed chondrogenic differentiation in response to bone morphogenetic protein 2 (BMP2) that is much more robust than control PDPCs (Figure 3A, Safranin O). Ext1-deficient pellets also displayed enhanced hypertrophic differentiation, as revealed by immunostaining for type $\mathrm{X}$ collagen (Figure 3A, Col10). Analysis of the expression of chondrogenic markers, namely Sox9, Col2a1, and Aggrecan (Acan), also confirmed enhanced chondrogenic differentiation of Ext1-deficient PDPCs (Figure 3B). Moreover, Ext1-deficient PDPCs exhibited greater levels of phosphorylation of Smad1/5/8 proteins in response to BMP2 than did control PDPCs (Figure 3C). This enhancement in Smad1/5/8 phosphorylation is not due to elevated expression of the BMP2 receptor BMPR1A in Ext1-deficient PDPCs (Figure 3C). Together, these results show that loss of HS expression enhances BMP signaling and chondrogenic differentiation in perichondrial progenitor cells.

BMP signaling is upregulated in the perichondrium of Ext1 mutant mice. To define the physiological significance of the above observations, we analyzed BMP signaling in the perichondrium of Col2a1-Ext1 ${ }^{\text {CKO }}$ mice. Immunostaining with anti-phospho-Smad1/5/8 (pSmad1/5/8) antibody revealed an expansion of the distribution of pSmad1/5/8-positive cells in the perichondrium of Col2a1-Ext ${ }^{\text {CKO }}$ mice. In controls, pSmad1/5/8-positive cells were localized mainly in the periosteal side of the perichondrium, while the cells in the groove of Ranvier were largely devoid of $\mathrm{pSmad} 1 / 5 / 8$ immunoreactivity (Figure $4, \mathrm{~A}$ and $\mathrm{B}$ ). In Col2a1-Ext $1^{\text {CKO }}$ mice, the distribution of $\mathrm{pSmad1/5/8-positive} \mathrm{cells} \mathrm{expanded} \mathrm{toward} \mathrm{the} \mathrm{epiphyseal} \mathrm{side,}$ and cells in the groove of Ranvier, including the cells in abnormal cell clusters, were immunoreactive for pSmad1/5/8 (Figure 4, A and B). Enhancement in pSmad1/5/8 expression was also observed in the rib perichondrium (Figure 4C). We also examined whether BMP signaling is enhanced in cells composing osteochondromas. Both the cartilage cap of discrete osteochondromas (Figure 4D) and the overgrown growth plate cartilage (Figure $4 \mathrm{E}$ ) were immunoreactive for $\mathrm{pSmad1/5/8}$. These results corroborate the data from PDPC cultures and support the model that loss of HS expression confers enhanced BMP signaling in the perichondrium, leading to osteochondromagenesis.

Pharmacological inhibition of BMP signaling suppresses osteochondroma development in vivo. To seek evidence for the causative role of BMP signaling in osteochondromagenesis, we examined whether pharmacological inhibition of BMP could suppress osteochondromagenesis. Among several small molecule BMP inhibitors, 
A
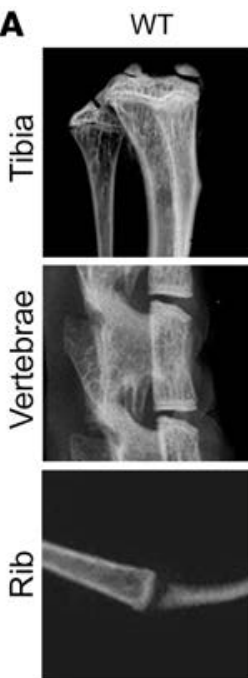

C
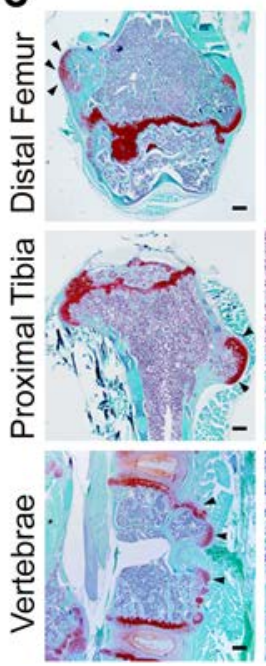
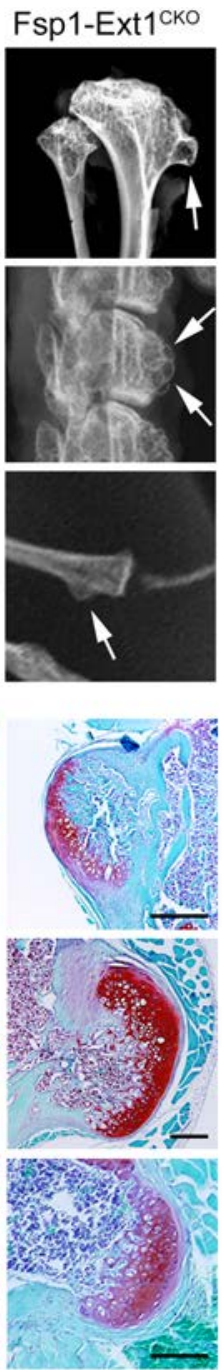

B

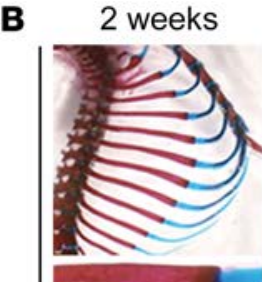

芫
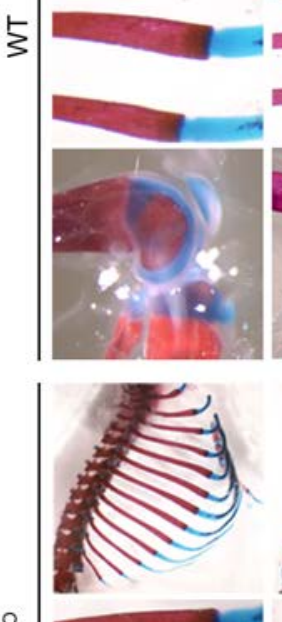

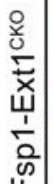

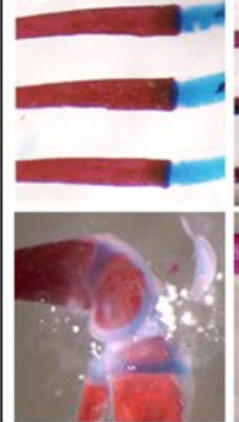

4 weeks

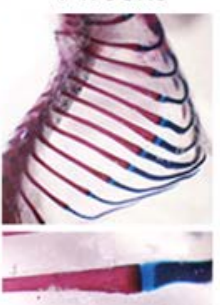

12 weeks
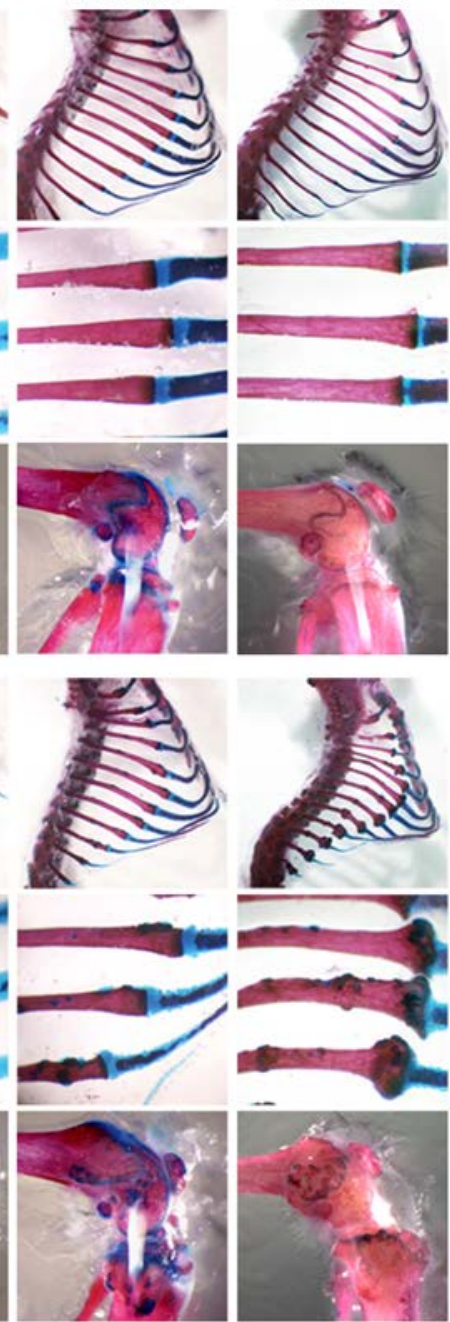

Figure 2. Perichondrium-targeted conditional Ext1-KO mice develop multiple osteochondromas. (A) X-ray images of 4-week-old Fsp1-Ext $7^{\text {CKO }}$ and control littermates (Ext flox/flox; WT). Fsp1-Ext ${ }^{\text {CKO }}$ mice show bony protrusions in long bones, vertebrae, and rib bones (arrows). (B) Whole-mount skeletal preparations of Fsp1-Ext1 ${ }^{C K 0}$ and control littermates (Ext $7^{\text {flox/flox; }}$ WT) at 2, 4, and 12 weeks of age. (C) Safranin O/Fast Green-stained sections through bony tumors in the femur, tibia, and vertebrae of Fsp1-Ext1 ${ }^{\mathrm{KKO}}$ mice (arrowheads). These tumors have a tissue structure consistent with osteochondroma (right panels). Scale bars: $0.05 \mathrm{~mm}$. Data shown are representative images; each analysis was performed on at least 3 animals per genotype.

we chose the inhibitor of type I BMP receptors LDN-193189 for this study because its pharmacological properties in mice, including efficacy and toxicity, have been extensively characterized (33, 34). Also, LDN-193189 has been shown to be effective in reducing heterotopic ossification in a FOP mouse model (33).

Col2a1-Ext $1^{\text {CKO }}$ mice were injected i.p. with $10 \mathrm{mg} / \mathrm{kg}$ LDN-193189 daily for 3 weeks starting at P7. This dosage of LDN-193189 had no apparent detrimental effects either on the overall growth or the longitudinal growth of the radius of the treated animals (Figure 5B). Treatment with LDN-193189 resulted in a significant decrease in the number of osteochondromas in rib bones (Figure 5, A and B). In the wrist joint area, the ectopic growth of growth plate cartilage between the radius and ulna, which mimics the "kissing exostoses" seen in human MHE, was significantly reduced (Figure 5, A-C). Another obvious change was the reduction of swelling in the chondro-osseous junction in rib bones (Figure 5C). Histologically, the disorganization of the cartilage-perichondrium boundary was not entirely normalized but was much less prominent in LDN193189-treated mice than controls (Figure 5C). To confirm that these changes are indeed induced by inhibition of BMP signaling, the level of pSmad1/5/8 expression was analyzed in the wrist joint area by IHC. 
A

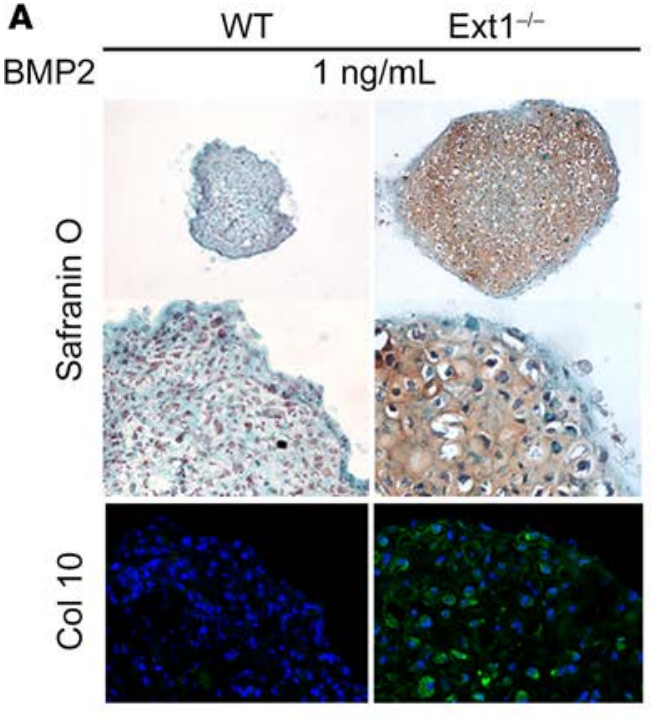

WT

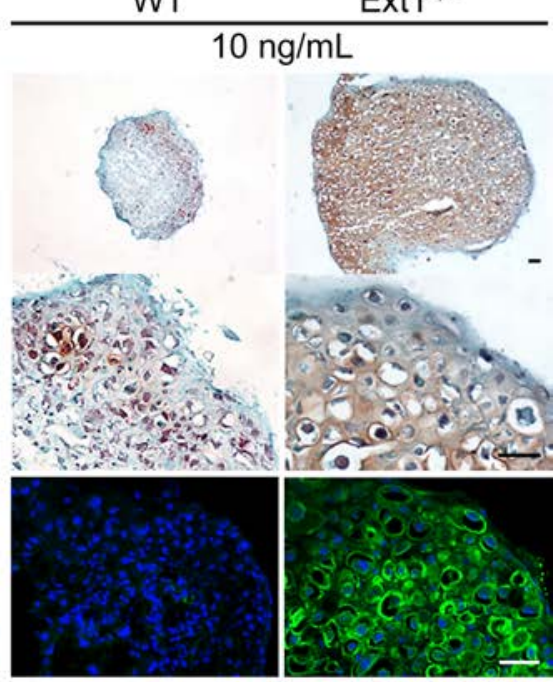

Col 10 / TO-PRO-3
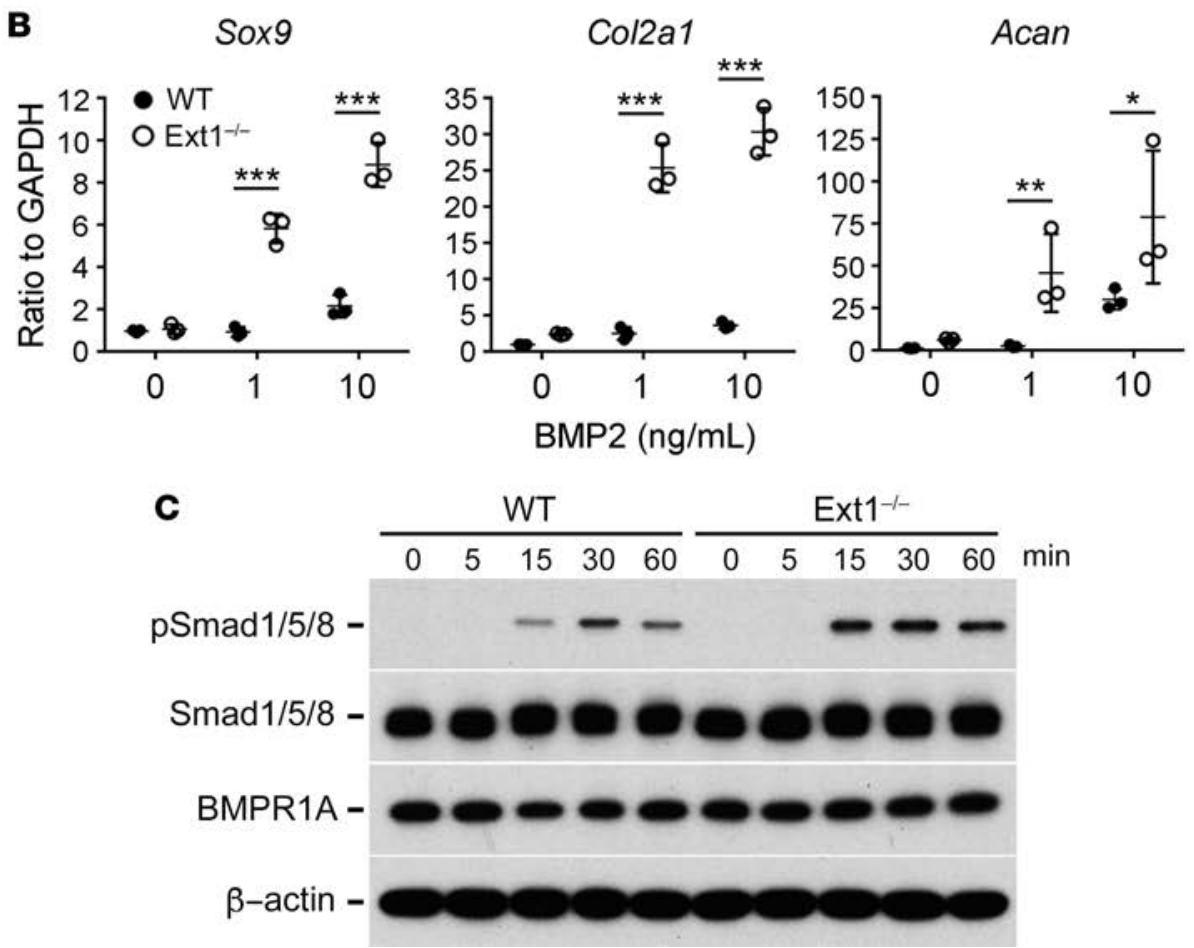

Figure 3. Ext1-deficient perichondrium-derived mesenchymal progenitor cells (PDPCs) exhibit enhanced chondrogenic differentiation and BMP signaling. (A and B) Ext1-deficient (Ext1 $\left.{ }^{-/}\right)$and WT (Ext flox/flox; WT) PDPCs were cultured as 3-dimensional cell pellets for 2 weeks in the presence of $1 \mathrm{ng} / \mathrm{ml}$ or $10 \mathrm{ng} / \mathrm{ml}$ of BMP2. (A) Safranin 0 staining and immunohistochemical staining of type X collagen (Col10) of pellets. Pellets derived from Ext1-deficient PDPCs are larger and more intensely stained with Safranin 0 and for type $X$ collagen than those derived from WT PDPCs. Scale bars: 0.1 mm. Data shown are representative of 3 independent experiments. (B) Expression of chondrogenic markers in pellet cultures derived from Ext1-deficient (Ext1 ${ }^{-1-}$ ) and WT (Ext $7^{f l o x}$ fflox; WT) PDPCs. Expression of Sox9, Col2a1, and Aggrecan (Acan) was evaluated by qPCR. Gapdh was used as an internal control for normalization. Means \pm SD $(n=3)$ are shown as horizontal bars. $P$ values were determined by two-way ANOVA. ${ }^{*} P<0.05,{ }^{* *} P<0.01,{ }^{* * *} P<0.001$. (C) Time course of BMP2-induced phosphorylation of Smad1/5/8. Ext1-deficient and WT PDPCs in monolayer cultures were stimulated with $10 \mathrm{ng} / \mathrm{ml} B M P 2$, and cells were lysed after indicated time of incubation. Cell lysates were immunoblotted with antibodies to phosphorylated Smad1/5/8, total Smad1/5/8, BMPR1A, and $\beta$-actin. This experiment was performed 3 times with similar results. 
A
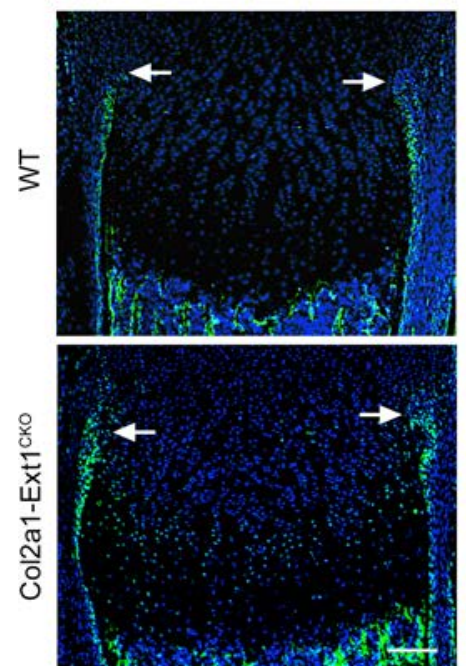

D

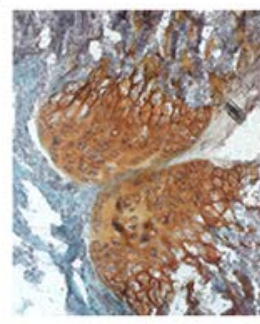

B
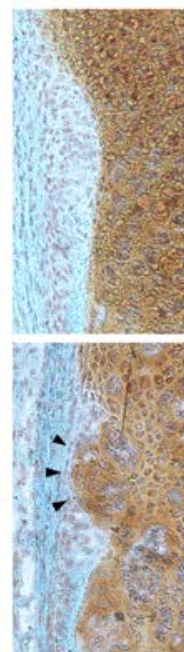
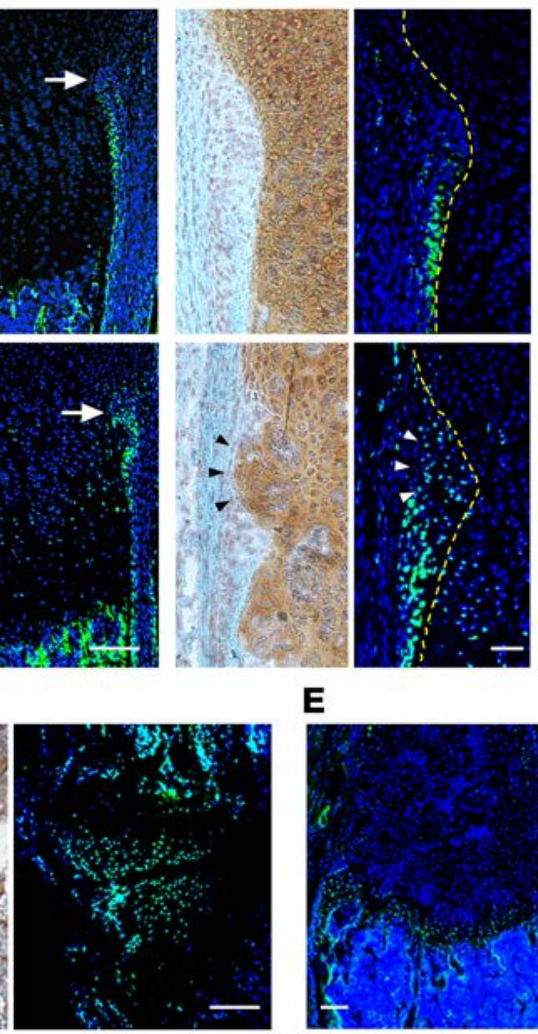

$\mathbf{E}$

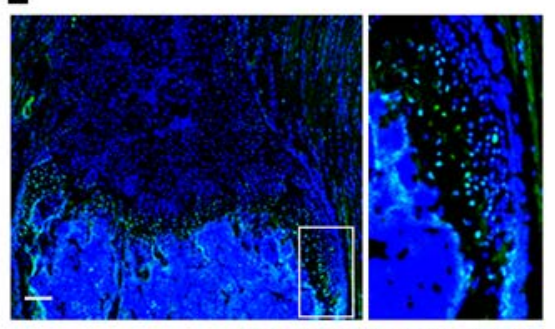

C
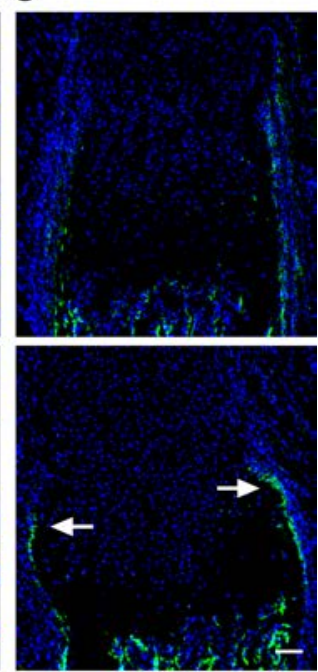

i

pSmad1/5/8 / TO-PRO-3

Figure 4. BMP signaling is upregulated in the perichondrium and osteochondromas of Col2a1-Ext ${ }^{\text {cko }}$ mice. (A-C) Frozen sections of the radius (A, B) and a rib bone (C) from P10 Col2a1-Ext7 $7^{c K O}$ and control (Ext $7^{f l o x}$ fflox; WT) littermates were double-labeled with anti-pSmad1/5/8 antibody (green) and TO-PRO-3 (blue) or stained with Safranin 0. (A) $\mathrm{pSmad1/5/8}$ expression in the perichondrium of the radius. The location of the groove of Ranvier is indicated by arrows. Note that the intensity and spatial extension of $\mathrm{pSmad1} / 5 / 8$ immunoreactivity is increased in the perichondrium of Col2a1-Ext $1^{\text {CKO }}$ mice. (B) High-power views of the groove of Ranvier. In control mice (Ext $1^{f l o x}$ flox), most pSmad1/5/8 immunoreactivity is seen in the diaphyseal side of the groove of Ranvier, and few pSmad1/5/8-immunoreactive cells are present within the groove per se. In Col2a1-Ext $7^{C K O}$ mice, the distribution of pSmad1/5/8-expressing cells is expanded both laterally and longitudinally. The cells forming the abnormal cell cluster within the groove of Ranvier (indicated by arrowheads) are also pSmad1/5/8-positive. Broken lines depict the perichondrium/growth plate boundary. (C) pSmad1/5/8 expression in the perichondrium of a rib bone. While WT perichondrium shows little pSmad1/5/8 immunoreactivity, perichondrium of Col2a1-Ext $7^{\mathrm{CKO}}$ mice displays strong pSmad1/5/8 immunoreactivity (arrows). (D) pSmad1/5/8 expression in osteochondromas formed in the forelimb of P28 Fsp1-Ext $7^{\mathrm{CKO} O}$ mouse. Adjacent sections were stained with Safranin 0 (left panel) or double-labeled with anti-pSmad1/5/8 antibody and TO-PRO-3 (right panel). Cells forming the cartilage cap of osteochondromas are pSmad1/5/8-positive. (E) pSmad1/5/8 expression in overgrown cartilage in a rib bone of P28 Fsp1-Ext $7^{C K O}$ mouse. The image on the right shows an enlarged view of the area indicated by a rectangle. Cells forming overgrown cartilage are immunoreactive to $\mathrm{pSmad1} / 5 / 8$. Scale bars: $0.1 \mathrm{~mm}(\mathbf{A}, \mathbf{C}, \mathbf{D}, \mathbf{E}) ; 20$ $\mu \mathrm{m}$ (B). Data shown are representative images; each analysis was performed on at least 3 mice per genotype.

pSmad1/5/8 immunoreactivity in the overgrown cartilage was decreased in LDN-193189-treated animals compared with control animals (Figure 5D), indicating that BMP signaling in the tissue that is relevant to osteochondromagenesis was attenuated by systemic administration of LDN-193189.

We further corroborated these findings by analyzing the Fsp1-Ext ${ }^{\text {CKO }}$ model. First, we confirmed by immunostaining with anti-pSmad1/5/8 antibody that BMP signaling is enhanced in the perichondrium of $F_{s p} 1$-Ext $1^{\text {CKO }}$ mice, as in Col2a1-Ext ${ }^{\text {CKO }}$ mice (Figure 6A). We then performed a second drug treatment study on Fsp1-Ext $1^{\text {CKO }}$ mice. Unlike Col2al-Ext $1^{1 K O}$ mice - many of which fail to thrive, dying after $\mathrm{P} 40-F s p 1-E x+1{ }^{C K O}$ mice can survive more than 1 year. Also, the onset of osteochondroma formation in Fsp1-Ext $1^{\mathrm{CKO}}$ mice is delayed relative to that in Col2a1-Ext ${ }^{C K O}$ mice. Thus, Fsp1-Ext ${ }^{C K O}$ mice were treated for 4 weeks starting at P14, instead of for 3 weeks starting at P7 in the case of Col2a1-Ext1 ${ }^{\text {CKO }}$ mice. As shown in Figure 6, B and C, treatment with LDN-193189 caused a pronounced decrease in the number of osteochondromas in rib bones. Unlike in the case of Col2a1-Ext $1^{\text {CKO }}$ mice, the growth of the radius in these mice was slightly inhibited, presumably due to the longer treatment duration, 
A
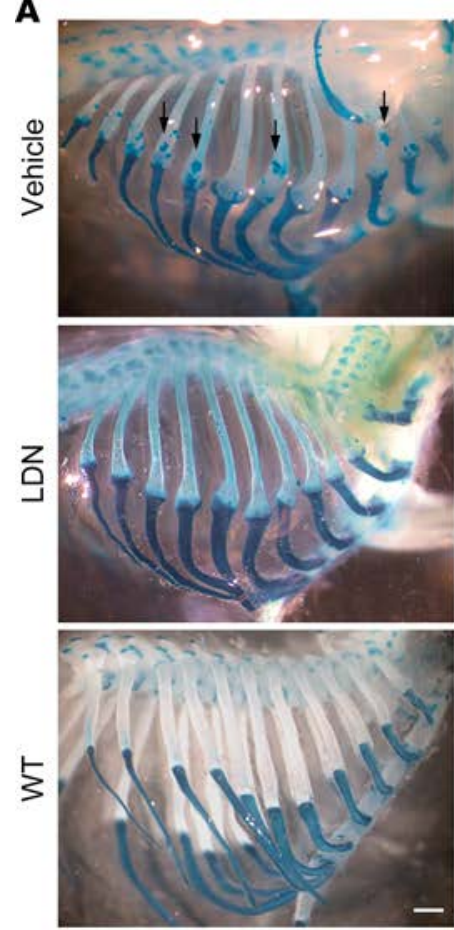

C
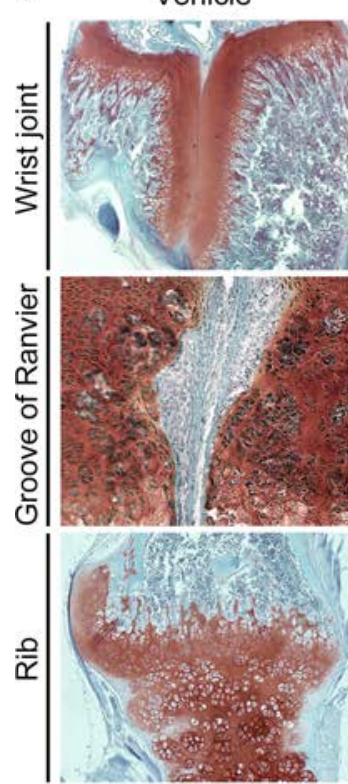
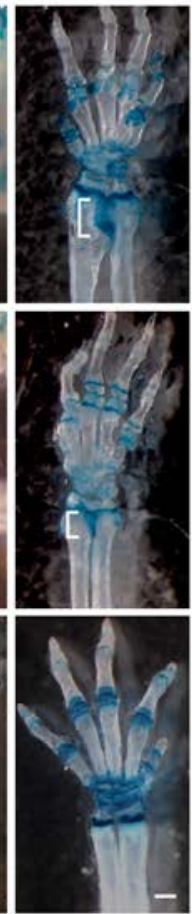

LDN
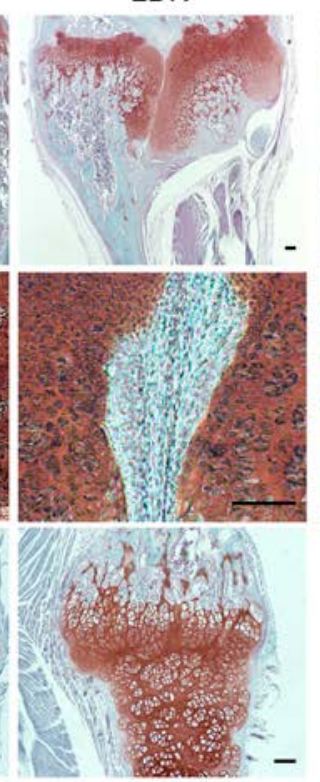

B
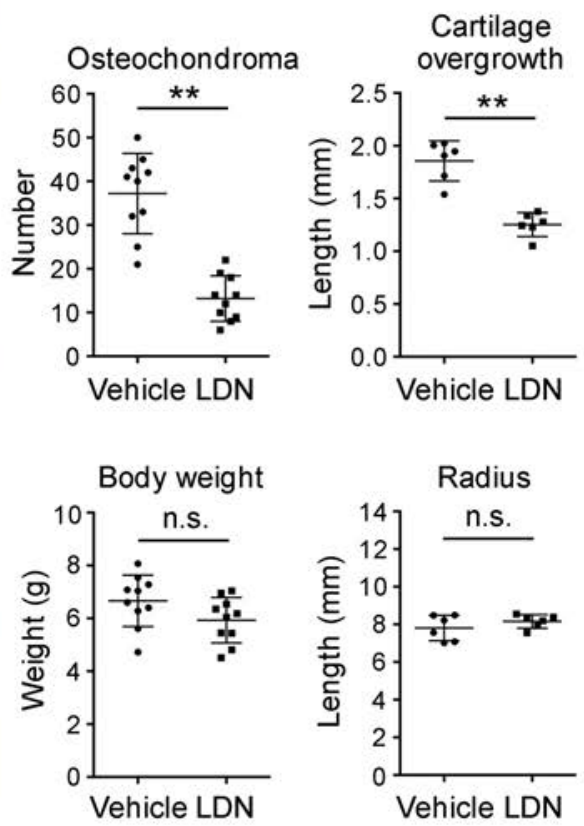

D
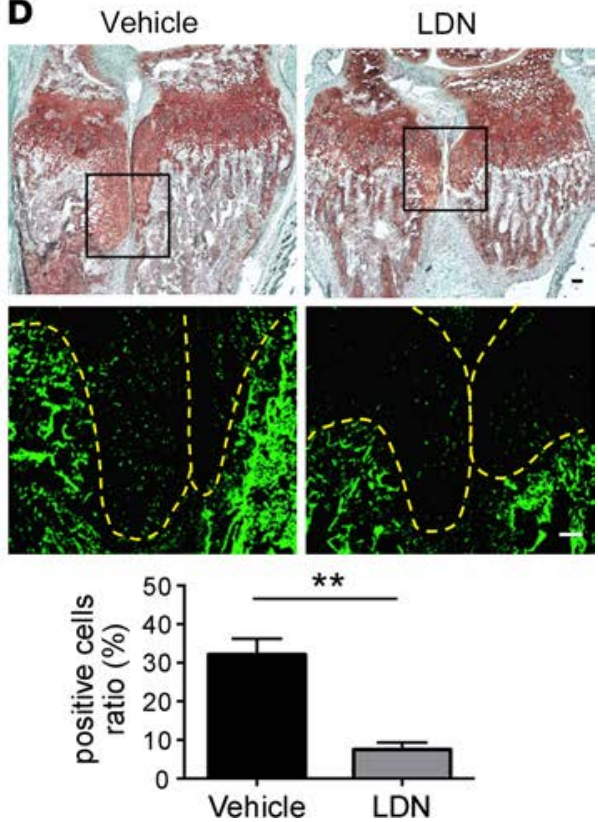

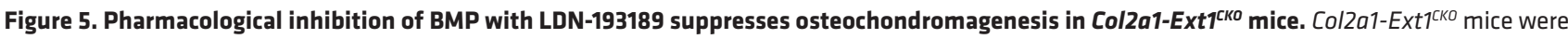
treated with LDN-193189 as described in Methods. (A) Whole-mount skeletal preparations of the rib cage and the forelimb of LDN-193189- and vehicle-treated mice. LDN-193189 suppresses osteochondroma formation in rib bones (arrows) and cartilage overgrowth in the wrist joint area (white brackets). Swelling of the chondro-osseous junction are also mitigated. Data shown are representative images of 10 animals per genotype. Specimens of WT control

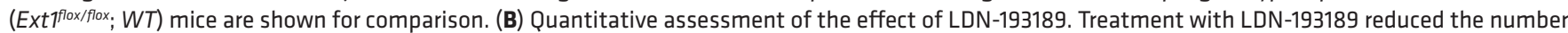
of osteochondromas in rib bones and the length of cartilage overgrowth between the radius and ulna. Body weight and the longitudinal growth of the radius were not affected. Means $\pm S D$ ( $n=10$ for the number of osteochondroma and body weight; $n=6$ for the size of cartilage overgrowth and the length of the radius) were shown as horizontal bars. ${ }^{* *} P<0.01$ by Student's $t$ test. (C) Safranin 0 -stained sections of the wrist joint area (top), the groove of Ranvier (middle), and the chondro-osseous junction of a rib bone (bottom). In LDN-193189-treated animals, the size of cartilage overgrowth between the radius and ulna is reduced (top panels) and the disorganization of perichondrium/cartilage boundaries in the groove of Ranvier and in the rib bone is reduced (middle and bottom panels). Representative images were obtained from at least 3 mice per genotype. (D) Effects of LDN-193189 on BMP signaling in situ. pSmad1/5/8 immunoreactivity in the overgrown cartilage (indicated by broken lines) is reduced in LDN-193189-treated animals compared with vehicle-treated animals. The bar graph shows the ratio of the number of pSmad1/5/8-immunoreactive cells relative to the total number of cells determined by TO-PRO-3 staining. Means $\pm \mathrm{SD}(n=8)$ are shown as horizontal bars. ${ }^{*} P<0.01$ by Student's $t$ test. The representative images were shown. Scale bars: $1 \mathrm{~mm}(\mathbf{A}) ; 0.1 \mathrm{~mm}(\mathbf{C}, \mathbf{D})$. 
while there was no difference in the body weight. Overall, these results demonstrate that inhibition of BMP signaling with LDN-193189 suppresses osteochondromagenesis in both Col2a1-Ext $1^{\text {CKO }}$ and Fsp1-Ext ${ }^{\text {CKO }}$ MHE mouse models, suggesting the possible therapeutic use of small molecule BMP inhibitors in MHE.

\section{Discussion}

This study is aimed at understanding how loss of HS due to inactivation of Ext1 leads to the development of multiple osteochondromas. Two unsolved issues are addressed in this study, namely the cellular origin of osteochondromas and the signaling aberration underlying osteochondromagenesis.

The cellular origin of osteochondoma in MHE, either from chondrocytes in the growth plate or from cells in the perichondrium, has been debated for some time (35). One reason for this debate is the difficulty of interrogating the initial phases of osteochondromagenesis using human osteochondroma specimens. Previous data using explant cultures of mouse long bone anlagen support the perichondrial origin of osteochondromas (12). Our observation of the presence of abnormal cell clusters in the perichondrial groove of Ranvier (Figure 1) also suggests that osteochondromas arise from progenitor cells residing in the perichondrium. In this study, we used perichondrium-targeted conditional $\mathrm{KO}$ to obtain direct genetic evidence for the perichondrial origin of osteochondromas. As shown in Figure 2, Fsp1-Ext $1^{C K O}$ mice developed multiple osteochondromas, confirming that osteochondromas can be derived from Ext1 inactivation in perichondrial cells. To corroborate this genetic observation, we also show that the loss of HS expression in perichondrium-derived mesenchymal progenitor cells promotes chondrogenic differentiation of these cells. Together with the observation that HS is strongly expressed in the perichondrium (Figure 1 and Supplemental Figure 1), these findings indicate that HS plays a critical role in the fate decision of perichondrial progenitor cells and that dysregulation of this process is the key defect that leads to osteochondromagenesis in MHE. It should be noted that our finding does not necessarily rule out the possibility that osteochondromas can also be derived from differentiated chondrocytes (11). However, our present study reveals that the expression of HS is much lower in growth plate chondrocytes than in perichondrium (Figure 1 and Supplemental Figure 1), and neither of our mouse models (Col2a1-Ext $1^{C K O}$ and Fsp1-Ext $1^{C K O}$ ) develops enchondroma-like intramedullary tumors. These observations argue against the chondrocytic origin of osteochondromas in these mouse models.

There have been several reports suggesting that aberrant BMP signaling plays a role in osteochondromagenesis $(12,27-30,36)$. This present study provides multiple lines of functional evidence for this hypothesis. First, Ext1-deficient PDPCs exhibit upregulation of BMP signaling and enhanced BMP-induced chondrogenic differentiation (Figure 3). Second, upregulation of BMP signaling is observed in the perichondrium of both Col2a1-Ext $1^{\text {CKO }}$ and Fsp1-Ext1 ${ }^{\text {CKO }}$ MHE models (Figure 4). Finally, pharmacological inhibition of BMP signaling by LDN-193189 suppresses osteochondromagenesis in these two MHE models (Figure 5). The inability of LDN-193189 to fully normalize MHE phenotypes may be due to nonoptimal dosage or to involvement of other BMP receptors in addition to ALK2 in osteochondromagenesis. It is also possible that HS-binding growth factors/morphogens other than BMPs — such as FGFs, Indian hedgehog homolog (IHH), and VEGF — are involved in osteochondromagenesis. Still, the present results support the notion that dysregulation of BMP signaling in the absence of HS is the primary molecular culprit that induces osteochondromagenesis in MHE.

It has been reported that loss of HS enhances, rather than suppresses, BMP signaling $(27,28,36)$. Our present study confirms this mode of action of HS on BMP signaling. This mode of action is distinct from that of HS in FGF signaling, in which HS proteoglycans (HSPGs) act as obligatory FGF coreceptors. As a result, loss of HS leads to suppression of FGF-mediated signaling. In contrast, BMP receptors do not require HSPG coreceptors for their interaction with BMPs. Endogenous HSPGs act as a sink to sequester BMPs and restrict their interaction with BMP receptors (28). Additionally, natural BMP antagonists, such as Noggin and Chordin, bind to and are potentiated by HS $(37,38)$. All these observations suggest a mechanistic model in which loss of HS leads to an increased availability of free BMPs for receptor activation, leading to enhanced BMP downstream signaling. Our results, both in vitro and in vivo, are consistent with this model.

The suppressive effect of LDN-193189 on osteochondroma formation not only provides functional evidence for the primary role of enhanced BMP signaling in osteochondromagenesis, but also suggests the possibility of pharmacological treatment for human MHE. In this study, we demonstrate the suppressive effect of LDN-193189 in two MHE models based on conditional KO of Ext1. While this paper was being revised, Sinha et al. (39) reported a similar effect of LDN-193189 on osteochondroma-like

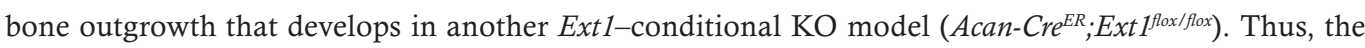
molecular mechanism of osteochondromagenesis shown in this paper appears to be applicable to at 
A

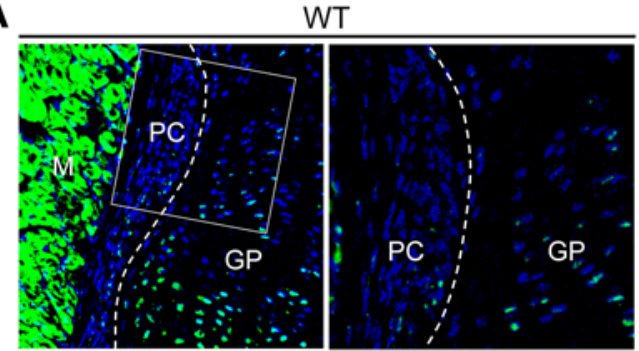

$\mathbf{B}$

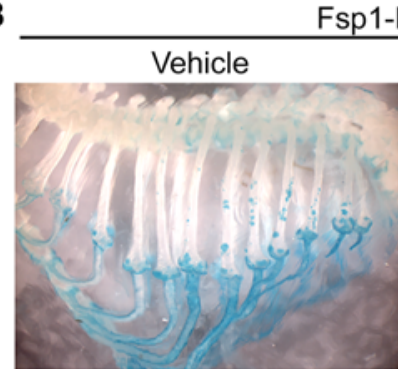

C

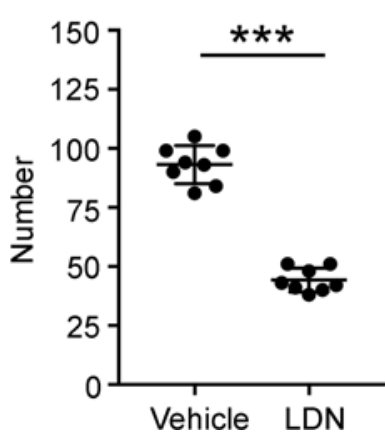

Fsp1-Ext1 ${ }^{\text {cko }}$

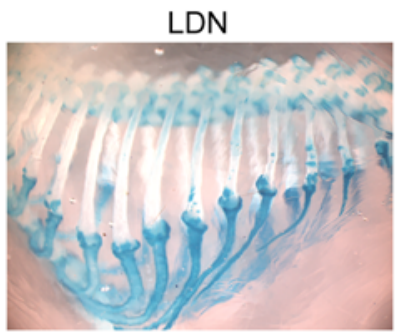

Radius

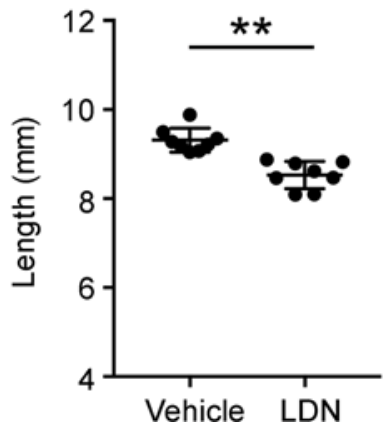

Fsp1-Ext1 ${ }^{\text {cko }}$

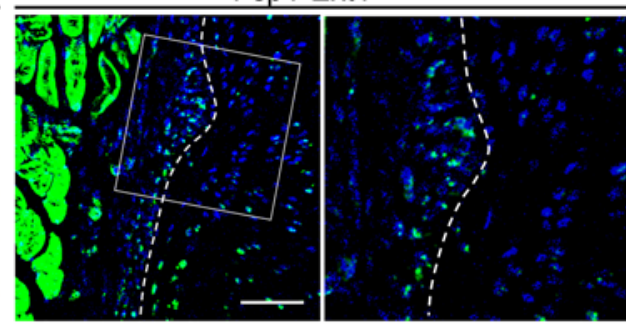

pSmad1/5/8 / TO-PRO-3

WT

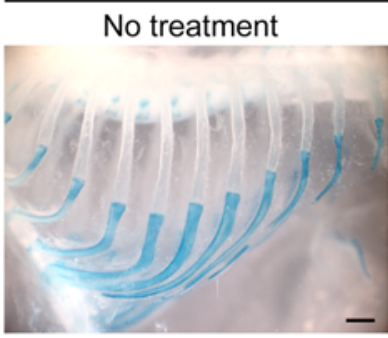

Body weight

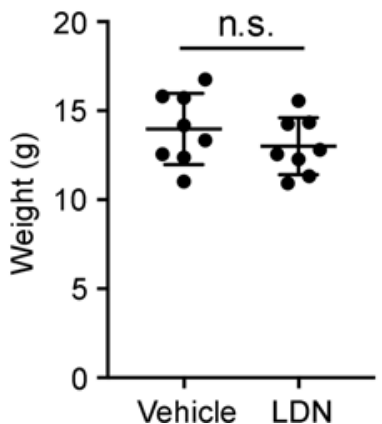

Figure 6. Role of aberrant BMP signaling in osteochondromagenesis in Fsp1-Ext1 ${ }^{\mathrm{CKO}}$ mice. (A) BMP signaling is upregulated in the perichondrium of Fsp1-Ext ${ }^{K K O}$ mice. Frozen sections of a rib bone from P14 Fsp1-Ext $1^{C K O}$ and control

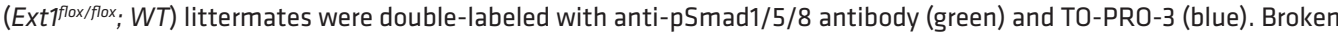
lines depict the perichondrium/growth plate boundary. Areas indicated by rectangles are enlarged in neighboring panels. PC, perichondrium; GP, growth plate; M, muscle. Scale bar: $0.1 \mathrm{~mm}$. (B and C) Effects of LDN-193189 treatment on osteochondromagenesis in Fsp1-Ext ${ }^{\kappa K O}$ mice. Fsp1-Ext ${ }^{\text {KKO }}$ mice were treated with LDN-193189 as described in Methods. (B) Representative images of whole-mount skeletal preparations of the rib cage of LDN-193189- and vehicle-treated mice. A preparation of a WT mouse is shown for comparison (WT). Scale bar: $1 \mathrm{~mm}$. (C) Quantitative assessment of the effect of LDN-193189 on the number of osteochondromas in rib bones, the length of the radius, and the body weight. Means \pm SD $(n=8)$ are shown as horizontal bars. ${ }^{* *} P<0.01,{ }^{* * *} P<0.001$ by Student's $t$ test.

least three MHE mouse models. An issue not explored in this study is the effect of LDN-193189 on

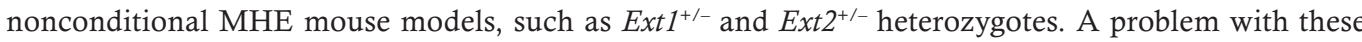
models is that, while they recapitulate the human MHE genotypes, their osteochondroma phenotype is noticeably different from that of human MHE, in that osteochondromas develop only in rib bones with a low penetrance $(6,40)$. This feature makes nonconditional MHE models less practical for preclinical drug treatment studies.

Currently, surgical resection of existing osteochondromas is essentially the only treatment for MHE (41). Since MHE patients tend to develop dozens of osteochondromas during the course of the disease, the burden of multiple surgeries is significant, and there is an acute need for pharmacological treatment to alleviate the number or the extent of osteochondromas. While our study used LDN-193189, as it has been extensively characterized in the context of FOP, new BMP inhibitors are being developed. With further refinement of efficacy and selectivity, small molecule BMP inhibitors may be promising therapeutic and/or preventive agents for MHE. 


\section{Methods}

Conditional KO mice. The loxP-modified Ext1 allele (Ext $\left.1^{f l o x}\right)$ was created as described previously (9). Mice carrying the Col2a1-Cre $E^{E R T}$ (8) and Fsp1-Cre (20) transgenes were obtained from Susan Mackem (National Cancer Institute, Frederick, Maryland, USA) and Eric Neilson (Northwestern University, Chicago, Illinois, USA), respectively. Rosa26-lacZ $(R 26 R)$ mice were obtained from The Jackson Laboratory. Col2a1-Ext ${ }^{\text {CKO }}$ mice were generated as described previously (10). Perichondrium-targeted conditional Ext1-KO mice were generated by crossing the Ext ffox $^{f l}$ allele and the Fsp1-Cre transgene. Genotyping of the mice was performed by PCR as described previously (10). All mouse lines used in this study had been backcrossed to C57B1/6J at least 10 generations.

Antibodies and regents. The following antibodies were used in this study: mouse monoclonal anti-type II collagen (catalog ab54236), rat polyclonal anti-CD44 (catalog ab25340), and rat monoclonal anti-Sca-1 (catalog ab51317) from Abcam; rabbit polyclonal anti-pSmad1/5/8 (catalog 9511) and rabbit monoclonal antiSox9 (catalog 82630) from Cell Signaling Technology; rabbit polyclonal anti-Smad1/5/8 (catalog sc-6031-R) from Santa Cruz Biotechnology Inc.; mouse monoclonal anti-HS (clones 10E4 and 3G10) from Seikagaku America; mouse monoclonal anti-Stro-1 (catalog 39-8401) from Invitrogen; rabbit polyclonal anti-type X collagen (catalog RDI-COLL10abr) from Fitzgerald; Alexa 488-labelled goat anti-rabbit IgG (catalog A-11034) and Alexa 594-labeled goat anti-mouse IgG (catalog A-11005) from Invitrogen; and horseradish peroxidaseconjugated goat anti-rabbit and goat anti-mouse IgG $(\mathrm{H}+\mathrm{L})$ from Bio-Rad. TO-PRO-3 and DAPI were purchased from Invitrogen.

Histology and IHC. Tissue specimens were fixed in 4\% paraformaldehyde (PFA) in PBS, decalcified in EDTA, embedded in paraffin, and sectioned at $5 \mu \mathrm{m}$ in thickness. For Safranin O/Fast Green staining, deparaffinized, rehydrated sections were stained in $0.02 \%$ aqueous Fast Green (Sigma-Aldrich), followed by a rinse in $1 \%$ acetic acid and $0.1 \%$ aqueous Safranin O (Sigma-Aldrich). For tartrate-resistant acid phosphatase (TRAP) staining, deparaffinized, rehydrated sections were stained with TRAP staining solution (Sigma-Aldrich). For frozen-section preparation, bones were harvested and immediately frozen in SCEM compound (Section-Lab). The cut surface was covered with an adhesive film (Cryofilm type IIC9, Section-Lab) and sections $(5 \mu \mathrm{m})$ were prepared with a microtome (Leica CM3050 S, Leica Microsystems) according to a method described previously $(42,43)$. For Safranin O/Fast Green staining, frozen sections were postfixed in $4 \%$ PFA in PBS and stained in $0.02 \%$ aqueous Fast Green, followed by a rinse in $1 \%$ acetic acid and $0.1 \%$ aqueous Safranin O. For immunostaining, frozen sections $(5 \mu \mathrm{m})$ were postfixed in $4 \%$ PFA and blocked with $5 \%$ goat serum $/ 0.3 \%$ Triton X-100 in PBS. Sections were incubated overnight at $4{ }^{\circ} \mathrm{C}$ with primary antibodies, followed by detection with secondary antibodies conjugated to the indicated fluorophores at concentrations recommended by manufacturers. Nuclei counterstaining was performed by TO-PRO-3 or DAPI. For immunostaining with mouse monoclonal antibodies, sections were additionally incubated with an unconjugated affinity purified $\mathrm{F}(\mathrm{ab})$ fragment goat anti-mouse IgG $(\mathrm{H}+\mathrm{L})$ (Jackson ImmunoResearch) overnight at $4^{\circ} \mathrm{C}$ before proceeding to the incubation with primary antibodies. For staining with the antiHS 10E4 antibody, sections were pretreated with chondroitinase ABC ( $2 \mathrm{U} / \mathrm{ml}$; Sigma-Aldrich) for 2 hours at $37^{\circ} \mathrm{C}$. Whole-skeleton X-gal staining was performed as described previously (10).

Analysis of the skeleton. For whole-mount analysis of skeletons, mice were eviscerated and fixed in $95 \%$ ethanol overnight. The preparations were stained with alcian blue for 1-3 days, rinsed in 95\% ethanol, and incubated in $2 \% \mathrm{KOH}$ for $24-48$ hours. Then they were stained with alizarin red S for 1-3 days. Stained preparations were cleaned in $20 \%$ glycerol $/ 1 \% \mathrm{KOH}$ for $5-14$ days and transferred to $50 \%$ glycerol/50\% ethanol for photography and storage. In some cases, skeletal preparations were stained without alizarin red S staining.

Isolation and characterization of PDPCs. Isolation of perichondrial progenitor cells was performed according to the method described for isolation of MSCs from the perichondrium of long bones (31). The entire rib cage was dissected from Ext $f^{f l o x / f l o x}$ mice at P1, and individual rib bones were cleaned free of muscles and connective tissues. The cleaned bones were incubated in $2 \mathrm{mg} / \mathrm{ml}$ collagenase (type 2, Worthington) in DMEM for 30 minutes at $37^{\circ} \mathrm{C}$, and then perichondrium was peeled off and triturated to dissociate cells. Dissociated cells were plated in DMEM containing 10\% FBS (Invitrogen), and adhesive cells were cultured for 2 weeks in the presence of $5 \mathrm{ng} / \mathrm{ml} \mathrm{FGF2} \mathrm{(BioVision)} \mathrm{and} 5 \mathrm{U} / \mathrm{ml}$ heparin (Sigma-Aldrich). Cells were then characterized by flow cytometry. For flow cytometry, cells were labeled using a Mouse Mesenchymal Stromal Cell Marker Panel (Abcam, catalog ab93759) and analyzed on a FACSCanto flow cytometer (BD Biosciences). Flow cytometric data were analyzed with FlowJo software (Tree Star Inc.).

Lentiviral transduction of cells. Cre-IRES-Puro lentivirus particles were produced from the Cre-IRESPuro plasmid (Addgene; plasmid 30205). Control IRES-Puro lentivirus particles were produced from a 
plasmid, in which the NLS-Cre sequence was deleted from Cre-IRES-Puro. Lentiviral particles were produced in the Viral Core facility at Sanford Burnham Prebys Medical Discovery Institute. For transduction, $2 \times 10^{6}$ PDPCs were seeded in a $10-\mathrm{cm}$ dish with lentiviral particles at a multiplicity of infection of 5 . After 24 hours, cells were fed with fresh medium and cultured for another 24 hours. Then, puromycin $(10 \mu \mathrm{g} / \mathrm{ml})$ was added to cultures, and cells were selected for 2 days. Cells were washed and fed with fresh medium for another 2 days before used in experiments.

Pellet cultures. PDPCs were detached by enzyme-free cell dissociation buffer (Gibco) and suspended in complete chondrogenic differentiating media consisting of DMEM (high glucose), $100 \mathrm{nM}$ dexamethasone, $50 \mu \mathrm{g} / \mathrm{ml}$ ascorbic acid-2-phosphate, $100 \mu \mathrm{g} / \mathrm{ml}$ sodium pyruvate, $40 \mu \mathrm{g} / \mathrm{ml} \mathrm{L}$-proline, and $1 \times \mathrm{ITS}+$ (Sigma-Aldrich). Aliquots of $2.0 \times 10^{5}$ cells in $1 \mathrm{ml}$ of defined media were spun down at $150 \mathrm{~g}$ for $5 \mathrm{~min}$ utes. Pellets were incubated with recombinant human BMP2 (Affymetrix, eBioscience) at $37^{\circ} \mathrm{C}$ for 14 days. For histological analyses, pellets were fixed in 4\% PFA/0.3\% sucrose in PBS, and then immersed in $30 \%$ sucrose. Fixed pellets were mounted in OCT embedding compound and frozen at $-80^{\circ} \mathrm{C}$ until use.

Immunoblotting. Cells were lysed in ice-cold $50 \mathrm{mM}$ Tris- $\mathrm{HCl}(\mathrm{pH} 7.5)$ containing $250 \mathrm{mM} \mathrm{NaCl}, 0.1 \%$ Triton X-100, $1 \mathrm{mM}$ EDTA, $50 \mathrm{mM} \mathrm{NaF}, 0.1 \mathrm{mM} \mathrm{Na}_{3} \mathrm{VO}_{4}, 1 \mathrm{mM}$ DTT, $0.1 \mathrm{mM}$ leupeptin, $0.1 \mu \mathrm{g} / \mathrm{ml}$ soybean trypsin inhibitor, $10 \mathrm{~g} / \mathrm{ml} \mathrm{L}-1$ chlor-3-(4-tosylamido)-4 phenyl-2-butanon (TPCK), $10 \mu \mathrm{g} / \mathrm{ml} \mathrm{L}$ 1 chlor-3-(4-tosylamido)-7-amino-2-heptanon-hydrochloride (TLCK), $10 \mu \mathrm{g} / \mathrm{ml}$ aprotinin, and $50 \mu \mathrm{g} / \mathrm{ml}$ phenylmethylsulfonyl fluoride (PMSF; Sigma-Aldrich). Lysed cells were incubated on ice for 30 minutes and then centrifuged at 20,000 $\mathrm{g}$ for 20 minutes at $4^{\circ} \mathrm{C}$ to isolate cell lysate. Lysate $(10 \mu \mathrm{g})$ was subjected to SDS-PAGE on $8 \%-16 \%$ Tris-glycine gels (Invitrogen), followed by electroblotting onto an Immobilon PVDF membrane (EMD Millipore). The ECL Western Blotting Substrate (Thermo Fisher Scientific) was used to detect signals.

Differentiation assays. Osteogenic, adipogenic, and chondrogenic differentiation of PDPCs were induced by culturing in the following differentiation-inducing media: Osteogenesis, 10\% FCS/DMEM containing $1 \mathrm{nM}$ dexamethasone, $20 \mu \mathrm{M} \beta$-glycerolphosphate, and $50 \mathrm{mM}$ ascorbic acid-2-phosphate; Adipogenesis, $10 \%$ FCS/DMEM containing $0.5 \mu \mathrm{M}$ dexamethasone, $0.5 \mu \mathrm{M}$ isobutyl-methylxanthine, and $50 \mu \mathrm{M}$ indomethacin; Chondrogenesis, DMEM containing $100 \mathrm{nM}$ dexamethasone, $50 \mu \mathrm{g} / \mathrm{ml}$ ascorbic acid-2phosphate, $100 \mu \mathrm{g} / \mathrm{ml}$ sodium pyruvate, $40 \mu \mathrm{g} / \mathrm{ml}$ L-proline, $1 \times$ ITS + , and $10 \mathrm{ng} / \mathrm{ml}$ recombinant mouse TGF- $\beta 1$ (Affymetrix, eBioscience). After culturing for respective periods, levels of osteogenic, adipogenic, and chondrogenic differentiation were evaluated by staining cultures with alizarin red $\mathrm{S}$, oil red $\mathrm{O}$, and alcian blue, respectively.

$q P C R$. Total RNA was extracted from cell monolayers or pellets using the RNeasy kit with DNase (Qiagen). cDNA was synthesized using SuperScript VILO Master Mix (Invitrogen), and qPCR was performed by using the FAST SYBR Green qPCR kit (Invitrogen) and primers shown in Supplemental Table 2. All values were normalized to Gapdh levels, and qPCR data were expressed as fold increases compared with controls. Specificity was tested by melting-curve analysis.

Drug treatment. Col2a1-Ext $1^{\text {CKO }}$ mice (10 mice per group) were treated by i.p. injection of LDN-193189 (Sigma-Aldrich) $(10 \mathrm{mg} / \mathrm{kg}$ body weight in distilled water) or vehicle (distilled water) daily, beginning at 7 days of age, for 3 weeks. At the end of drug treatment, animals were sacrificed and processed to prepare whole-mount skeletal preparation. Assessment of the skeletal gross abnormalities and osteochondromagenesis were performed in the whole-mount skeletal preparations by an observer blinded to the type of treatment. The number of osteochondromas was determined by counting discrete osteochondromas in all rib bones of a whole-mount skeletal preparation under a stereoscopic microscope. The longitudinal length of overgrown cartilage between the radius and ulna, as well as the length of the radius, was measured with ImageJ software on the microphotographs taken by a CCD camera. For Fsp1-Ext ${ }^{\text {CKO }}$ mice ( 8 mice per group), LDN-193189 (10 mg/kg body weight in distilled water) or vehicle (distilled water) were i.p. injected daily beginning at 14 days of age for 4 weeks. At the end of drug treatment, animals were sacrificed and their skeletal preparations examined as described above.

Statistics. No statistical method was used to predetermine sample size. Statistical analyses were performed with GraphPad Prism 5. Student's two-sided $t$ test and two-way ANOVA were used under the assumption of normal distribution and observance of similar variance. $P<0.05$ was considered significant. Bonferroni post hoc analysis was performed where applicable. Values are expressed as mean \pm SD. For all of these experiments, between-group variances were similar and data were symmetrically distributed. Data shown are representative images; each analysis was performed on at least 3 mice per genotype. Immunos- 
taining was performed at least in triplicate. For other experiments, the numbers of biological replicates, animals, or cells are indicated in the text. There was no randomized allocation during the experiments and outcome assessment.

Study approval. All procedures were performed in accordance with NIH Guide for the care and use of Laboratory Animals (National Academies Press, 2011) and approved by the IACUC of the Sanford Burnham Prebys Medical Discovery Institute.

\section{Author contributions}

YY designed research; TI, SN, KM, and FI performed research; TI, FI, and YY analyzed data; TI and YY wrote the paper.

\section{Acknowledgments}

This work was supported by NIH grants R01AR055670 and R01AR062692 (to YY). TI was the recipient of a Postdoctoral Fellowship for Research Abroad from Japan Society for the Promotion of Science. SN was the recipient of Uehara Memorial Foundation Fellowship. We thank Susan Mackem and Eric Neilson for providing Col2a1-Cre ${ }^{E R T}$ and Fsp1-Cre mice, respectively; Daniel Legault-Coutu (Helmholtz Zentrum München) for providing a protocol and help for culturing perichondrial progenitor cells; and the MHE Research Foundation for support and encouragement.

Address correspondence to: Yu Yamaguchi, Sanford Burnham Prebys Medical Discovery Institute, 10901 North Torrey Pines Road, La Jolla, California 92037, USA. Phone: 858.646.3124; Email: yyamaguchi@ sbpdiscovery.org.

1. Bishop JR, Schuksz M, Esko JD. Heparan sulphate proteoglycans fine-tune mammalian physiology. Nature. 2007;446(7139):1030-1037.

2. Schmale GA, Conrad EU, Raskind WH. The natural history of hereditary multiple exostoses. J Bone Joint Surg Am. 1994;76(7):986-992.

3. McCormick C, et al. The putative tumour suppressor EXT1 alters the expression of cell-surface heparan sulfate. Nat Genet. 1998;19(2):158-161

4. Lind T, Tufaro F, McCormick C, Lindahl U, Lidholt K. The putative tumor suppressors EXT1 and EXT2 are glycosyltransferases required for the biosynthesis of heparan sulfate. J Biol Chem. 1998;273(41):26265-26268.

5. Senay C, et al. The EXT1/EXT2 tumor suppressors: catalytic activities and role in heparan sulfate biosynthesis. EMBO Rep. 2000;1(3):282-286.

6. Stickens D, Zak BM, Rougier N, Esko JD, Werb Z. Mice deficient in Ext2 lack heparan sulfate and develop exostoses. Development. 2005;132(22):5055-5068.

7. Stieber JR, Dormans JP. Manifestations of hereditary multiple exostoses. J Am Acad Orthop Surg. 2005;13(2):110-120.

8. Nakamura E, Nguyen MT, Mackem S. Kinetics of tamoxifen-regulated Cre activity in mice using a cartilage-specific CreER(T) to assay temporal activity windows along the proximodistal limb skeleton. Dev Dyn. 2006;235(9):2603-2612.

9. Inatani M, Irie F, Plump AS, Tessier-Lavigne M, Yamaguchi Y. Mammalian brain morphogenesis and midline axon guidance require heparan sulfate. Science. 2003;302(5647):1044-1046.

10. Matsumoto K, Irie F, Mackem S, Yamaguchi Y. A mouse model of chondrocyte-specific somatic mutation reveals a role for Ext1 loss of heterozygosity in multiple hereditary exostoses. Proc Natl Acad Sci USA. 2010;107(24):10932-10937.

11. Jones KB, et al. A mouse model of osteochondromagenesis from clonal inactivation of Ext1 in chondrocytes. Proc Natl Acad Sci USA. 2010;107(5):2054-2059.

12. Huegel J, et al. Perichondrium phenotype and border function are regulated by Ext1 and heparan sulfate in developing long bones: a mechanism likely deranged in Hereditary Multiple Exostoses. Dev Biol. 2013;377(1):100-112.

13. Zak BM, et al. Compound heterozygous loss of Ext1 and Ext2 is sufficient for formation of multiple exostoses in mouse ribs and long bones. Bone. 2011;48(5):979-987.

14. Mansoor A, Beals RK. Multiple exostosis: a short study of abnormalities near the growth plate. J Pediatr Orthop B. 2007; 16(5):363-365.

15. Karlsson C, Thornemo M, Henriksson HB, Lindahl A. Identification of a stem cell niche in the zone of Ranvier within the knee joint. J Anat. 2009;215(3):355-363.

16. Koyama E, Shimazu A, Leatherman JL, Golden EB, Nah HD, Pacifici M. Expression of syndecan-3 and tenascin-C: possible involvement in periosteum development. J Orthop Res. 1996;14(3):403-412.

17. David G, Bai XM, Van der Schueren B, Cassiman JJ, Van den Berghe H. Developmental changes in heparan sulfate expression: in situ detection with mAbs. J Cell Biol. 1992;119(4):961-975.

18. Long F, Chung UI, Ohba S, McMahon J, Kronenberg HM, McMahon AP. Ihh signaling is directly required for the osteoblast lineage in the endochondral skeleton. Development. 2004;131(6):1309-1318.

19. Ono N, Ono W, Nagasawa T, Kronenberg HM. A subset of chondrogenic cells provides early mesenchymal progenitors in growing bones. Nat Cell Biol. 2014;16(12):1157-1167.

20. Bhowmick NA, et al. TGF-beta signaling in fibroblasts modulates the oncogenic potential of adjacent epithelia. Science. 
2004;303(5659):848-851.

21. Strutz F, et al. Identification and characterization of a fibroblast marker: FSP1. J Cell Biol. 1995;130(2):393-405

22. Ebralidze A, et al. Isolation and characterization of a gene specifically expressed in different metastatic cells and whose deduced gene product has a high degree of homology to a Ca2+-binding protein family. Genes Dev. 1989;3(7):1086-1093.

23. Duarte WR, et al. S100A4: a novel negative regulator of mineralization and osteoblast differentiation. J Bone Miner Res. 2003;18(3):493-501.

24. Klingelhöfer J, Ambartsumian NS, Lukanidin EM. Expression of the metastasis-associated mts1 gene during mouse development. Dev Dyn. 1997;210(2):87-95.

25. Bowen ME, Ayturk UM, Kurek KC, Yang W, Warman ML. SHP2 regulates chondrocyte terminal differentiation, growth plate architecture and skeletal cell fates. PLoS Genet. 2014;10(5):e1004364.

26. Sgariglia F, et al. Epiphyseal abnormalities, trabecular bone loss and articular chondrocyte hypertrophy develop in the long bones of postnatal Ext1-deficient mice. Bone. 2013;57(1):220-231.

27. Jiao X, Billings PC, O'Connell MP, Kaplan FS, Shore EM, Glaser DL. Heparan sulfate proteoglycans (HSPGs) modulate BMP2 osteogenic bioactivity in C2C12 cells. J Biol Chem. 2007;282(2):1080-1086.

28. Fisher MC, Li Y, Seghatoleslami MR, Dealy CN, Kosher RA. Heparan sulfate proteoglycans including syndecan-3 modulate BMP activity during limb cartilage differentiation. Matrix Biol. 2006;25(1):27-39.

29. Kobayashi T, Lyons KM, McMahon AP, Kronenberg HM. BMP signaling stimulates cellular differentiation at multiple steps during cartilage development. Proc Natl Acad Sci USA. 2005;102(50):18023-18027.

30. Deirmengian GK, Hebela NM, O'Connell M, Glaser DL, Shore EM, Kaplan FS. Proximal tibial osteochondromas in patients with fibrodysplasia ossificans progressiva. J Bone Joint Surg Am. 2008;90(2):366-374.

31. Coutu DL, François M, Galipeau J. Inhibition of cellular senescence by developmentally regulated FGF receptors in mesenchymal stem cells. Blood. 2011;117(25):6801-6812

32. Arai F, Ohneda O, Miyamoto T, Zhang XQ, Suda T. Mesenchymal stem cells in perichondrium express activated leukocyte cell adhesion molecule and participate in bone marrow formation. J Exp Med. 2002;195(12):1549-1563.

33. Yu PB, et al. BMP type I receptor inhibition reduces heterotopic [corrected] ossification. Nat Med. 2008;14(12):1363-1369.

34. Boergermann JH, Kopf J, Yu PB, Knaus P. Dorsomorphin and LDN-193189 inhibit BMP-mediated Smad, p38 and Akt signalling in C2C12 cells. Int J Biochem Cell Biol. 2010;42(11):1802-1807.

35. Jochmann K, Bachvarova V, Vortkamp A. Heparan sulfate as a regulator of endochondral ossification and osteochondroma development. Matrix Biol. 2014;34:55-63.

36. Matsumoto Y, Matsumoto K, Irie F, Fukushi J, Stallcup WB, Yamaguchi Y. Conditional ablation of the heparan sulfate-synthesizing enzyme Ext1 leads to dysregulation of bone morphogenic protein signaling and severe skeletal defects. J Biol Chem. 2010;285(25):19227-19234.

37. Paine-Saunders S, Viviano BL, Economides AN, Saunders S. Heparan sulfate proteoglycans retain Noggin at the cell surface: a potential mechanism for shaping bone morphogenetic protein gradients. J Biol Chem. 2002;277(3):2089-2096.

38. Jasuja R, Allen BL, Pappano WN, Rapraeger AC, Greenspan DS. Cell-surface heparan sulfate proteoglycans potentiate chordin antagonism of bone morphogenetic protein signaling and are necessary for cellular uptake of chordin. J Biol Chem. 2004;279(49):51289-51297.

39. Sinha S, et al. Unsuspected osteochondroma-like outgrowths in the cranial base of Hereditary Multiple Exostoses patients and modeling and treatment with a BMP antagonist in mice. PLoS Genet. 2017;13(4):e1006742.

40. Hilton MJ, Gutiérrez L, Martinez DA, Wells DE. EXT1 regulates chondrocyte proliferation and differentiation during endochondral bone development. Bone. 2005;36(3):379-386.

41. Kitsoulis P, et al. Osteochondromas: review of the clinical, radiological and pathological features. In Vivo. 2008;22(5):633-646.

42. Kawamoto T, Shimizu M. A method for preparing 2- to 50-micron-thick fresh-frozen sections of large samples and undecalcified hard tissues. Histochem Cell Biol. 2000;113(5):331-339.

43. Arima Y, et al. Regional neural activation defines a gateway for autoreactive $\mathrm{T}$ cells to cross the blood-brain barrier. Cell. 2012;148(3):447-457. 